\title{
Percolation on hyperbolic lattices
}

\author{
Seung Ki Baek, ${ }^{1, *}$ Petter Minnhagen, ${ }^{1,+}$ and Beom Jun Kim ${ }^{2,+}$ \\ ${ }^{1}$ Department of Theoretical Physics, \\ Umeå University, 90187 Umeå, Sweden \\ ${ }^{2}$ Department of Physics, BK21 Physics Research Division, \\ Sungkyunkwan University, Suwon 440-746, Korea
}

\begin{abstract}
The percolation transitions on hyperbolic lattices are investigated numerically using finite-size scaling methods. The existence of two distinct percolation thresholds is verified. At the lower threshold, an unbounded cluster appears and reaches from the middle to the boundary. This transition is of the same type and has the same finite-size scaling properties as the corresponding transition for the Cayley tree. At the upper threshold, on the other hand, a single unbounded cluster forms which overwhelms all the others and occupies a finite fraction of the volume as well as of the boundary connections. The finite-size scaling properties for this upper threshold are different from those of the Cayley tree and two of the critical exponents are obtained. The results suggest that the percolation transition for the hyperbolic lattices forms a universality class of its own.

PACS numbers: 64.60.ah, 02.40.Ky, 05.70.Fh
\end{abstract}

\footnotetext{
*Electronic address: garuda@tp.umu.se

${ }^{\dagger}$ Electronic address: Petter.Minnhagen@physics.umu.se

${ }^{\ddagger}$ Electronic address: beomjun@skku.edu
} 


\section{INTRODUCTION}

Geometry is often crucial in physical phenomena, since dimensionality and topological defects determine the properties of phase transitions. Progress in complex networks is an example where geometrical features are paramount to the physical properties (for a review, see, e.g., Ref. [1]). This is also reflected in ongoing research interests in the nontrivial lattice structures such as fractal lattices [2] and Apollonian networks [3]. Another important area is the hyperbolic geometry where even the familiar standard physical models like electronic spins and Brownian motions exhibit novel behaviors [4]. Such studies are interesting not only from a theoretical viewpoint but also from a potential real applicability related to the rapid development of fabrication of devices and structures in nanoscales [5]. In this work, we focus on the percolation problem and investigate the percolation phase transition for negatively curved hyperbolic lattices. Percolation on hyperbolic lattices has so far been of predominantly mathematical interest and several interesting mathematical results have been reported, including the existence of an intermediate phase with infinitely many unbounded clusters [6, 7, 8]. More specifically, there exist in general two critical thresholds: unbounded clusters are formed at the first threshold, while unbounded clusters are merged to become one unique unbounded cluster at the second threshold. In case of the usual flat lattices, which have vanishing surface-volume ratios in infinite-volume limit, the two thresholds coincide, and the theory of such percolation transitions is well-developed [9]. On the other hand, the properties of the percolation transitions for hyperbolic lattices still remain to be further clarified. In particular, these lattices are not homogeneous due to the presence of a boundary and as a consequence, the critical properties may differ from the mean-field-type transitions which were discussed in Ref. [10]. In this paper, we investigate the characteristic features of the thresholds and the corresponding phases by using various statistical measures like the number of boundary points connected to the middle, the ratio of the first and second largest clusters, and the cluster size distribution. We use finite-size scaling methods to obtain the critical properties, together with the Newman-Ziff algorithm [11].

This paper is organized as follows: In Sec. II, we introduce alternative manifestations of the percolation thresholds and show that all of them coincide in an ordinary square lattice. In Sec. III, we explain the notion of a hyperbolic lattice and the appearance of two distinct percolation transitions. We start with the Bethe lattice and the standard mean-field results. 
Next the Cayley tree is introduced which is the simplest model with two thresholds. This model is used as a benchmark when discussing the characteristic features of percolation in hyperbolic lattices. The efficiency of the finite-size scaling methods is also illustrated for this simpler model. Then we present the results for the general hyperbolic structures which contain loops. We summarize our results in Sec. IV]

\section{VARIOUS MANIFESTATIONS OF PERCOLATION}

There are various possible manifestations of percolation. We here describe the critical thresholds corresponding to them as well as their relationship. The percolation threshold is usually defined as the occupation probability $p$ above which a cluster is formed which occupies a finite fraction of the system. Let us first consider an $L \times L$ square lattice. In this case it is well-known that $p_{c}=1 / 2$ for bond percolation [9]. Alternatively, we may consider the ratio between the largest cluster size, $s_{1}$, and the second largest one, $s_{2}$ [12]. This measure is based on the fact that above the transition threshold, the second largest cluster becomes negligible with respect to the largest one, and accordingly, as soon as $p$ exceeds a critical value from below, $s_{2} / s_{1}$ vanishes in the large lattice limit. Since it implies that one large unique cluster dominates the whole system, we call this threshold $p_{u}$. Figure 1(a) illustrates that the ensemble average of $s_{2} / s_{1}$ scales quite well with sizes for the square lattice. This finite-size scaling supports the well-known result that $p_{u}=p_{c}=1 / 2$ and the critical index $\nu=4 / 3$. We will use this type of finite-size scaling methods throughout the paper.

An alternative manifestation of percolation is the number $b$ of boundary points connected to the middle of the lattice via occupied bonds. We will also use this concept of midpoint percolation throughout the paper. Around the threshold of the midpoint percolation, which we will call $p_{m}$, a cluster penetrates the whole system and one expects a finite-size scaling of the form

$$
b=L^{\kappa} f\left[\left(p-p_{m}\right) L^{1 / \nu}\right]
$$

with $\kappa=\nu /(1+\nu)=4 / 7 \approx 0.57$ [13, 14]. Again, Fig. 1)(b) shows an excellent scaling collapse with $\kappa=4 / 7$ and $p_{m}=p_{c}=1 / 2$. Consequently, the finite size scaling of $b$ gives a practical alternative way of investigating percolation properties. Yet another measure, based on the same midpoint percolation concept, is the fraction of the boundary points connected 

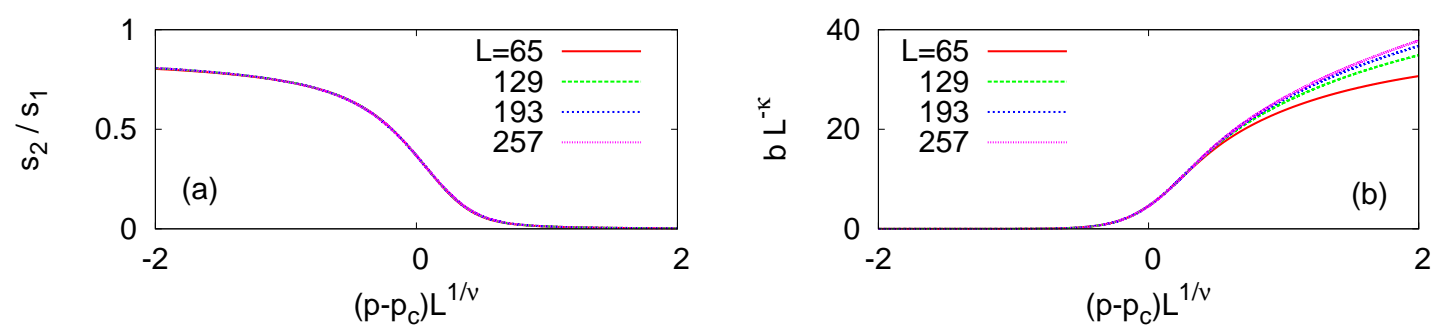

FIG. 1: (Color online) Scaling plots for regular square lattices, using (a) the ratio between the largest and the second largest cluster sizes, and (b) the number of boundary points connected to the midpoint of the lattice. The two-dimensional percolation scaling exponent $\nu=4 / 3$ and $\kappa=\nu /(1+\nu)=4 / 7$ are used for scaling collapse with $p_{u}=p_{c}=1 / 2$. The average is taken over $10^{6}$ independent realizations for each plot.

TABLE I: Percolation thresholds and quantities used for their numerical detections. Here $s_{i}$ represents the $i$ th largest cluster size, $b$ is the number of boundary points connected to the middle of the lattice, and $B$ is the total number of boundary points.

\begin{tabular}{|c|c|c|}
\hline \hline Threshold & Meaning & Observable \\
\hline$p_{u}$ & The unbounded cluster becomes unique. & $s_{2} / s_{1}$ \\
$p_{m}$ & The boundary is connected to the middle. & $b$ \\
$p_{b}$ & A finite fraction of the boundary is connected to the middle. & $b / B$ \\
\hline \hline
\end{tabular}

to the middle, $b / 4 L$, which becomes finite when $p$ gets above $p_{b}$. This measure will be also frequently used. For the planar lattices all these thresholds coincide so that we have only one critical threshold $p_{c}=p_{u}=p_{m}=p_{b}=1 / 2$. (The observable quantities used in the present paper are listed in Table I.) The crucial point in the present context is that this equality does not hold for hyperbolic lattices.

\section{PERCOLATION IN HYPERBOLIC LATTICES}

Suppose that $n$ regular $m$-gons meet at every vertex, which we represent by a Schläfli symbol $\{m, n\}$ [15]. The resulting lattices are flat if we take $\{m, n\}=\{3,6\},\{4,4\},\{6,3\}$, which together constitute the two-dimensional (2D) percolation universality class [9]. If $(m-2)(n-2)>4$, on the other hand, the resulting lattice is known to have a constant 


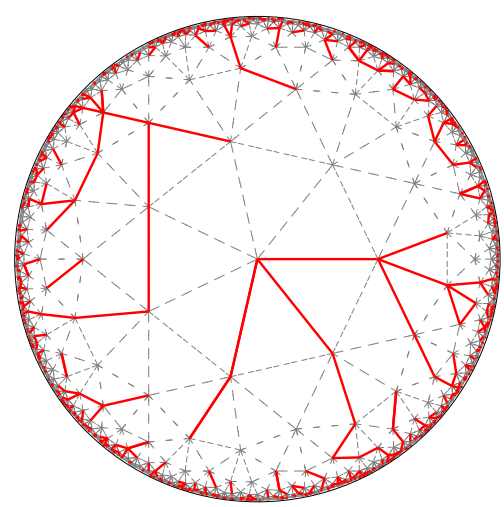

FIG. 2: (Color online) Poincaré disk representation of the hyperbolic lattice $\{m, n\}=\{3,7\}$ [seven $(n=7)$ triangles $(m=3)$ meet at every vertex] with the total number of layers $l=6$. Solid lines show occupied bonds in a realization with the occupation probability of $p=0.2$.

negative Gaussian curvature [16], making a hyperbolic surface. Figure 2 shows a hyperbolic lattice represented as $\{3,7\}$, where seven regular triangles meet at each vertex. Note that all of the triangles in this figure are congruent with respect to the metric used in this projection on the Poincaré disk [17]. As shown in Fig. 2, we construct the lattice in a concentric way so that the origin of the disk becomes the zeroth layer and its seven nearest neighbors constitute the first layer. Likewise, the second layer surrounding the first layer is composed of 21 vertices, and so on up to the $l$ th layer $[l=6$ in Fig. 2]. The system is said to have a level $l$, and its size is given by $N(l)=1+7 \sum_{j=1}^{l}\left[c_{+}^{j}-c_{-}^{j}\right] / \sqrt{5}$ with $c_{ \pm}=(3 \pm \sqrt{5}) / 2$. For example, $N(l=2)=1+7 \frac{\left(c_{+}-c_{-}\right)}{\sqrt{5}}+7 \frac{\left(c_{+}^{2}-c_{-}^{2}\right)}{\sqrt{5}}=1+7+21=29$. This formula shows that the number of lattice points increases exponentially with a distance from the origin $O$, yielding a nonvanishing surface-volume ratio in the limit $N \rightarrow \infty$. In other words, the total number of boundary points $B$ is proportional to the system size $N$. The sizes of other lattices with different Schläfli symbols are listed in Table II.

Since a $d$-dimensional hypercube with a volume $v$ has the surface-volume ratio $\propto v^{-1 / d}$, a hyperbolic lattice is usually called infinite-dimensional. In graph theory, an object with a nonvanishing surface-volume ratio is called nonamenable [7, 8]. The exponential increase of lattice points as a function of the distance from the middle also constitute a practical challenge since the range of possible sizes is limited when using the finite-size scaling method. For this reason, we present numeric values only up to the second digit in this work. 
TABLE II: System sizes for various structures, $\{m, n\}$, as a function of level $l$. Each lattice is grown up from a single midpoint in the zeroth layer.

\begin{tabular}{|c|c|}
\hline \hline$\{m, n\}$ & $N(l)$ \\
\hline$\{3,7\}$ & $1+\frac{7}{\sqrt{5}} \sum_{j=1}^{l}\left[\left(\frac{3+\sqrt{5}}{2}\right)^{j}-\left(\frac{3-\sqrt{5}}{2}\right)^{j}\right]$ \\
$\{4,5\}$ & $1+\frac{5}{\sqrt{3}} \sum_{j=1}^{l}\left[(2+\sqrt{3})^{j}-(2-\sqrt{3})^{j}\right]$ \\
$\{5,5\}$ & $1+\sqrt{5} \sum_{j=1}^{l}\left[\left(\frac{7+3 \sqrt{5}}{2}\right)^{j}-\left(\frac{7-3 \sqrt{5}}{2}\right)^{j}\right]$ \\
$\{6,4\}$ & $1+2 \sqrt{2} \sum_{j=1}^{l}\left[(3+2 \sqrt{2})^{j}-(3-2 \sqrt{2})^{j}\right]$ \\
$\{7,3\}$ & $1+\frac{15}{\sqrt{5}} \sum_{j=1}^{l}\left[\left(\frac{3+\sqrt{5}}{2}\right)^{j}-\left(\frac{3-\sqrt{5}}{2}\right)^{j}\right]$ \\
$\{\infty, 3\}$ & $1+3 \sum_{j=1}^{l} 2^{j-1}$ \\
\hline
\end{tabular}

\section{A. Bethe lattice}

The binary Bethe lattice is the infinite binary tree where all the lattice points are equivalent [18]. This means that the Bethe lattice lacks boundary points. Consequently, it belongs to the amenable class. This is in contrast to the Cayley tree (discussed in the following section) which includes the boundary even in the large lattice limit and hence is an example of a nonamenable graph. The percolation for the Bethe lattice is exactly solvable and the solution corresponds to the standard mean-field theory [9]. This standard mean-field theory describes the percolation transition for $d$-dimensional Euclidean lattices provided $d \geq 6[9]$. However, it has limited applicability in the context of nonamenable graphs.

The critical threshold $p_{c}$ is well-known since the early percolation theory formulated in the gelation process [19]. The point is that percolation on a Bethe lattice can be treated as the Galton-Watson branching process [20]. We pick up an arbitrary point as a root, and the set of all the points reached from it by $i$ bonds is called the $i$ th generation (the term generation will be used in trees, instead of layer). Let us denote $w$ as the extinction probability that the branching process from the root is ended at some finite generation of the tree. For such a process, each bond to the next generation should be either unoccupied with a probability $1-p$, or occupied but eventually terminated with the probability $p w$. Since each vertex has two bonds to the next generation, the sum of those probabilities has 
to be squared and $w$ satisfies a self-consistency equation, $w=(1-p+p w)^{2}$, yielding

$$
w= \begin{cases}1 & \text { for } 0 \leq p<1 / 2 \\ (1 / p-1)^{2} & \text { for } 1 / 2 \leq p \leq 1\end{cases}
$$

When $p>1 / 2$, the extinction probability is less than unity. From the percolation viewpoint, every vertex has one successor on average at $p=1 / 2$, and accordingly, the cluster from the root vertex can be extended indefinitely. Consequently, the bond percolation of the Bethe lattice has $p_{c}=1 / 2$, at which unbounded clusters may be formed. For a general Bethe lattice denoted as $\{\infty, n\}$, this generalizes into $p_{c}=1 /(n-1)$.

An important point in the present context is that amenable graphs only have one percolation threshold. Thus for Bethe lattice the threshold $p_{m}$ [where a cluster from the midpoint (which in this case is any point because all points are equivalent) reaches a point arbitrarily far away] and $p_{c}$ [the threshold where the unbounded clusters contain a finite fraction of the whole system] are equal, i.e., $p_{m}=p_{c}$ due to the equivalence between points. Also the three planar lattices $(\{4,4\},\{3,6\},\{6,3\})$ are amenable and consequently have only one threshold (compare with Sec. III). As will be discussed in the following, nonamenable graphs such as hyperbolic lattices have two distinct percolation thresholds.

\section{B. Cayley tree, $\{\infty, 3\}$}

The Cayley tree is a tree grown from the root vertex up to the $l$ th generation where the root vertex is identified as the middle of the lattice. This is an example of a nonamenable graph. While it has sometimes been presumed that the Bethe lattice is an adequate limiting case of the Cayley tree, more recent studies suggest that the Cayley tree in the limit of $l \rightarrow \infty$ has different critical properties from those of the Bethe lattice [1]. Note that vertices are not equivalent for the Cayley tree so that one can clearly define which generation a vertex belongs to. The branching-process argument is again applicable and the cluster from the root vertex reaches the bottom of the tree at $p_{m}=1 / 2$. On the other hand, the uniqueness

threshold is located at $p_{u}=1$ [6], since $s_{2} / s_{1}$ remains finite at any $p<1$ [Fig. 3(a)]. The vanishing of $s_{2} / s_{1}$ as $p_{u}=1$ is approached can be obtained as follows: The total number of bonds in the tree with a level $l$ is $K=2^{l+1}-2$. Suppose that precisely one bond is broken on average. The occupation probability corresponding to this is $p=(K-1) / K$. Suppose 
further that the broken bond connects the $i$ th and $(i+1)$ th generations. The probability to select this bond is given by

$$
P(i)=\frac{2^{i+1}}{2^{l+1}-2} .
$$

Breaking one bond creates precisely two clusters. The smaller one is below the broken bond and has a size of $s_{2}=2^{l-i}-1$. The size of the larger one is consequently $s_{1}=N-s_{2}=$ $2^{l+1}-2^{l-i}$. The expectation value of the ratio $s_{2} / s_{1}$ is obtained as follows:

$$
\begin{aligned}
\left\langle\frac{s_{2}}{s_{1}}\right\rangle & =\sum_{i=0}^{l-1} \frac{s_{2}}{s_{1}} P(i) \\
& =\sum_{i=0}^{l-1} \frac{2^{l-i}-1}{2^{l+1}-2^{l-i}} \frac{2^{i+1}}{2^{l+1}-2} \\
& =\sum_{i=0}^{l-1} \frac{2^{l-i}-1}{2^{l+1}-2^{l-i}} \frac{2^{i+1}}{2^{l+1}-2} \\
& \approx \frac{1}{2^{l+1}} \sum_{i=0}^{l-1} \frac{1}{1-2^{-i-1}} \\
& \approx \frac{l}{2^{l+1}}
\end{aligned}
$$

Since $p=(K-1) / K$, one can express this result directly in terms of $p$. By using the connection $1-p=K^{-1} \approx 2^{-l-1}$, one obtains

$$
\left\langle\frac{s_{2}}{s_{1}}\right\rangle \propto-(1-p) \log (1-p) .
$$

Thus the ratio $s_{2} / s_{1}$ vanishes as $-(1-p) \log (1-p)$ as the threshold $p_{u}=1$ is approached from below. This is illustrated in Fig. 3(a): The Cayley tree has two thresholds and $p_{m}<p_{u}$.

The midpoint percolation quantity $b$ instead possesses the scaling form

$$
b=l^{\kappa} f\left[\left(p-p_{m}\right) l^{1 / \nu}\right]
$$

with $\kappa=0$ and $\nu=1$. This scaling form can be derived as follows: The number of boundary points which is reached from the midpoint for a given value $p$ is $b=3 p \times(2 p)^{l-1}$ from which it follows that

$$
b=\frac{3}{2} \exp [\log (2 p) l] \approx \frac{3}{2} \exp \left[2 l\left(p-p_{m}\right)\right] .
$$

The validity of this finite-size scaling is demonstrated in [Fig. 3(b)] together with the exact scaling form given by Eq. (3). 

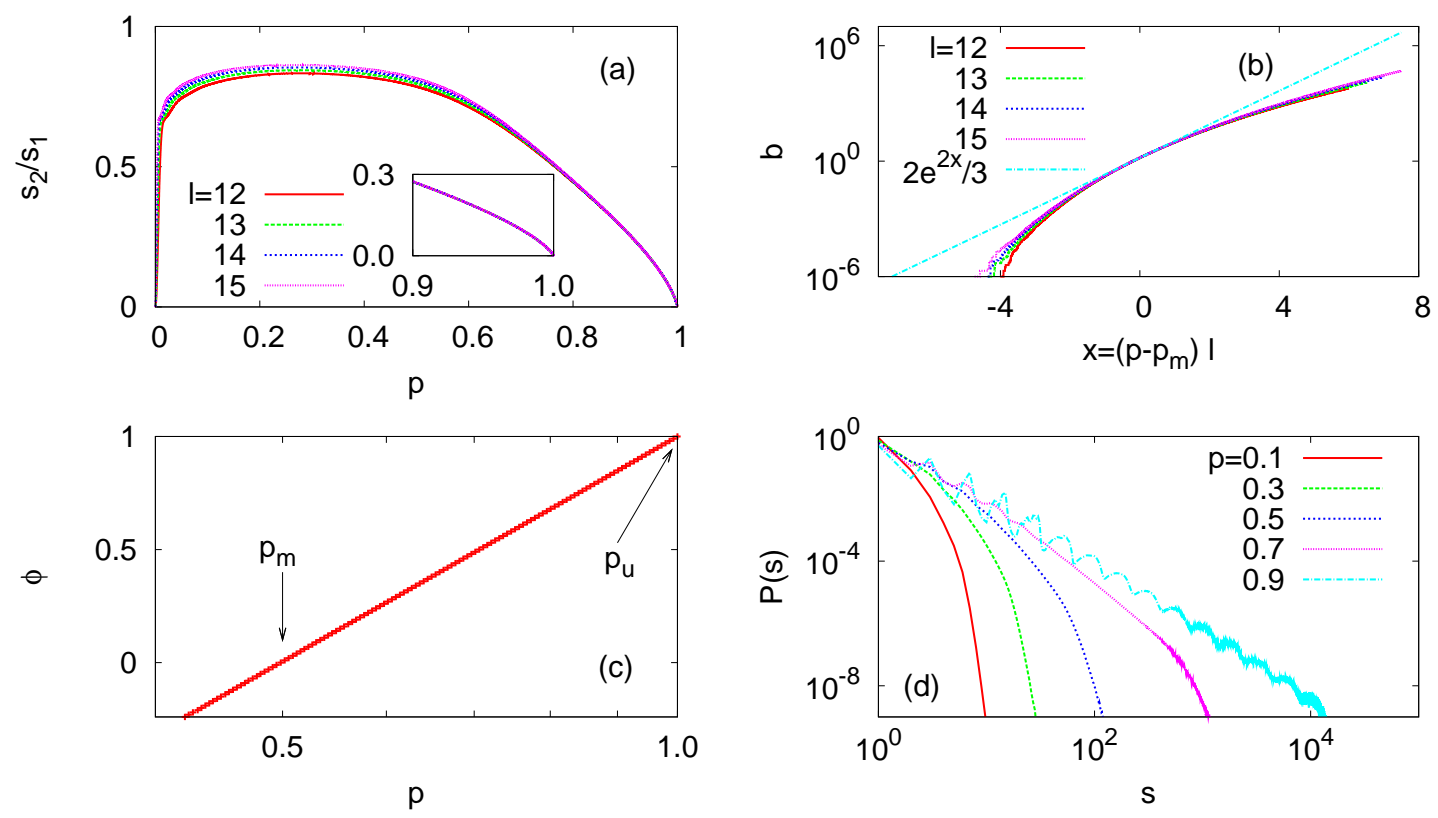

FIG. 3: (Color online) Numerical results in Cayley trees, averaged over $10^{6}$ realizations. (a) The ratio between $s_{2}$ and $s_{1}$. Inset: A closer look near $p=1$, where one can hardly see any size dependency. (b) Scaling plot by Eq. (2). The scaling function is well-described by $\frac{3}{2} \exp \left[2 l\left(p-p_{m}\right)\right]$ near $p_{m}=0.50$. (c) $\phi$ as a function of $p$, provided that $b(p) \sim B^{\phi}$. The horizontal axis is in the $\log$ scale, confirming $\phi=1+\log _{2} p$. (d) Cluster size distribution at various $p$ with $l=15$. It approaches to $P(s) \sim s^{-2}$ as $p \rightarrow 1$, while finiteness of the system adds an exponential cutoff.

One may also note that since $l \approx \log _{2} N$, it follows that $b \sim(2 p)^{l}=N p^{l} \approx N p^{\log _{2}{ }^{N}}=$

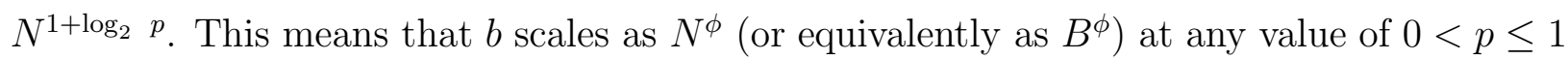
with a $p$-dependent exponent $\phi=1+\log _{2} p$ [Fig. 3(c)]. A direct consequence of this is that $b / B$ eventually goes to zero at every $p<1$ in the limit of infinite $N$. Thus $b / B$ is discontinuous at $p_{b}=1$ for $l=\infty$. This is in contrast to the ratio $s_{2} / s_{1}$ which goes continuously to zero at $p_{u}=1$.

One can also obtain the limiting behavior of the cluster size distribution $P(s)$ by removing one bond. In this case $P(s) d s$ is the probability of finding a cluster with a size in the interval $[s, s+d s]$ and $P(i) d i$ is the probabililty of the single bond to be broken between generations $i$ and $(i+1)$. This latter probability is also equal to the probability of finding a second 
largest cluster of size $s_{2} \propto s^{L-i}$. Thus $P\left(s_{2}\right) d s_{2}=P(i) d i$ from which follows that

$$
P\left(s_{2}\right)=P(i)\left|\frac{d i}{d s_{2}}\right| \propto s_{2}^{-2},
$$

where the last proportionality is obtained from $s_{2} \propto s^{L-i}$. This suggests that the size distribution of clusters $P(s)$ (excluding the largest one) should approach the form $P(s) \propto s^{-\tau}$ where $\tau \rightarrow 2$ as $p \rightarrow 1$. Since the generation $i$ in a tree is actually a discrete variable it follows that the possible sizes of $s_{2}$ are also discrete and this discreteness becomes noticeable as $p \rightarrow 1$. This is illustrated in Fig. 3(d) which shows the approach to $P(s) \propto s^{-2}$ as well as the log-periodic oscillations caused by the discreteness.

A more intuitive explanation for $P(s)$ may be gained by mapping the percolation problem in a Cayley tree to a branching process, namely, the formation of family trees [21]: Let us consider the family-size distribution in the $z$-ary tree $(z \equiv n-1)$ at the $k$ th generation, with the net birth rate $\lambda=\log (z p)$ as each family grows by $(z p)^{k}$. When a bond is broken in a tree graph, the top of this detached branch is interpreted as the first ancestor of a new family, and we describe this top vertex as an immigrant, whose number is proportional to the existing population size $N_{k}$. If the number of immigrants is written as $\zeta N_{k}$, the birth and immigration should yield a constant growth of population, $\zeta+\lambda=\log z$, as we know $N_{k+1}=z N_{k}$. According to Ref. [21], the family size distribution at the $k$-th generation exhibits a power law with an exponent $\tau^{\prime}=2+\zeta / \lambda=2+\log (1 / p) / \log (z p)$ as $k \rightarrow \infty$. Since the volume of a given tree is proportional to its surface, it seems plausible that $\tau \approx \tau^{\prime}$ for the cluster size distribution, $P(s)$. Note that $\tau^{\prime}=2$ for $p=1$ which is also the limiting result for the Cayley tree. This gives a hand-waving argument suggesting that the size distribution form $P(s) \propto s^{-\tau}$ could be valid also for some range of $p$ below one.

\section{Heptagonal lattice, $\{7,3\}$}

Now we consider a heptagonal lattice, denoted as $\{m, n\}=\{7,3\}$. Since the probability to find a loop is roughly $O\left(p^{m}\right)$, we expect the results obtained for a Cayley tree will remain valid to some extent. The lower threshold is determined from the finite-size scaling of $b$ and Eq. (21) as shown in Fig. 4(a). This determines the value of $p_{m} \approx 0.53$. We note that this is rather close to the exact result for the Cayley tree $1 /(n-1)=1 / 2$, and shows the same scaling form as in Eq. (2). This suggests that the loops have only a small effect on 

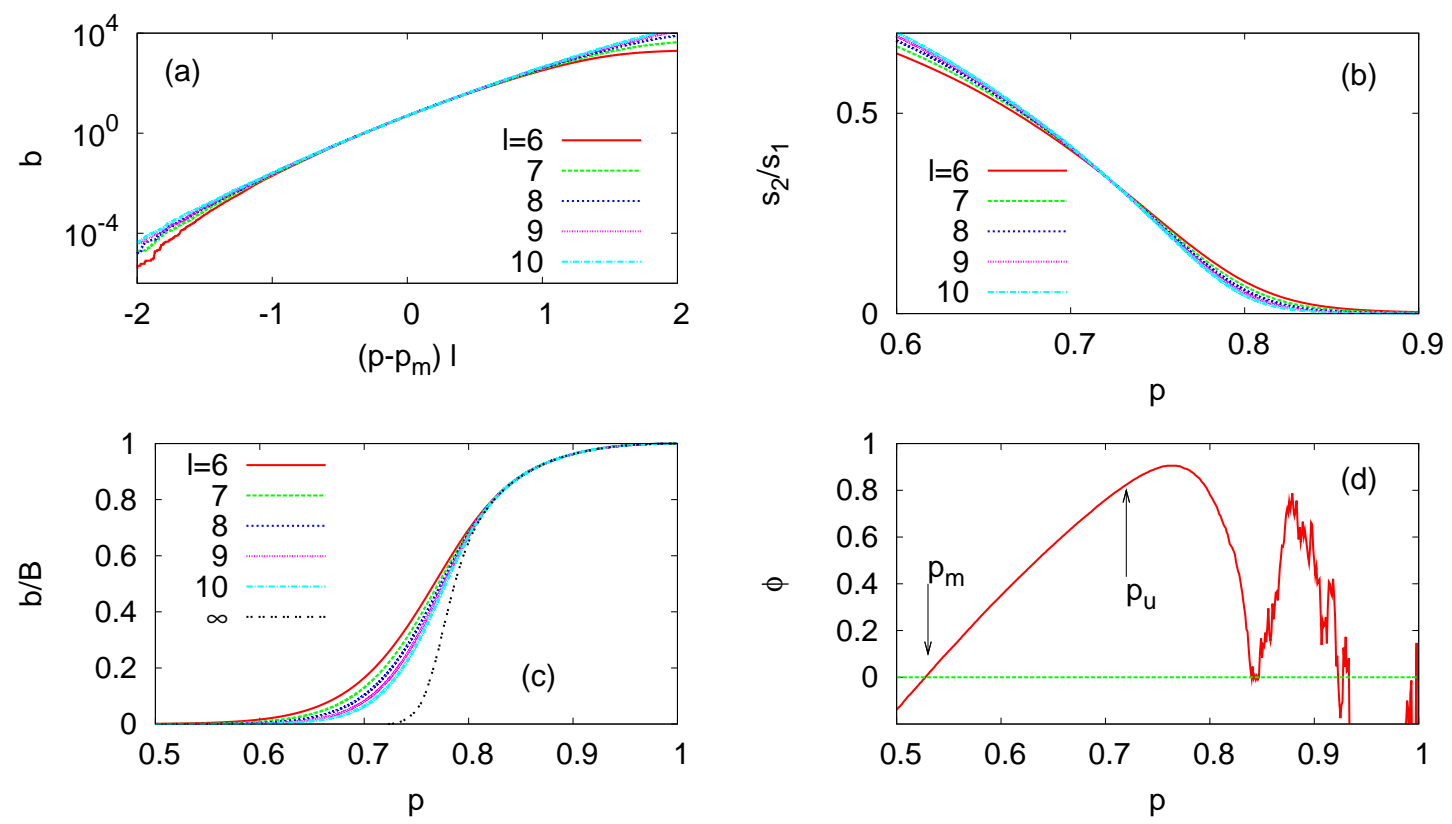

FIG. 4: (Color online) Numerical results for heptagonal lattices, $\{7,3\}$, averaged over $10^{6}$ realizations. (a) Measurement of $b$ yields a consistent result with the tree case. (b) The ratio between two largest cluster sizes $s_{2} / s_{1}$ gives $p_{u} \approx 0.72$, and (c) extrapolating $b / B$ gives $p_{b} \approx 0.72$, supporting $p_{b}=p_{u}$. (d) The exponent $\phi$ as a function of $p$ shows a clear difference from the tree case [see Fig. [3(c), for comparison]. At $p \gtrsim 0.84, \phi$ largely fluctuates as it is hard to determine the size dependency from the numerical data.

the percolation properties at small values of $p$, as is also suggested by the actual structure of the clusters illustrated in Fig. 2,

The upper threshold is determined from the finite-size scaling of $s_{2} / s_{1}$ which gives $p_{u} \approx$ 0.72. According to a dual-lattice argument [22], a percolation threshold for a regular lattice $\{m, n\}$ is predicted approximately as $m /(m+n)$. Applying this argument somewhat ad hoc to the present case yields $7 /(7+3)=0.7$, which is in fact fairly close to the actual value, $p_{u} \approx 0.72$.

Alternatively, the upper threshold can be determined from $b / B$ by assuming that the size scaling form is the same as for the Cayley tree,

$$
b / B \sim c_{1} N^{\phi-1}+c_{2}
$$

and extrapolating the numerical results from $l=6, \ldots, 11$ to the infinite-size limit [Fig. 4(c)]. This extrapolation gives $|b / B| \lesssim O\left(10^{-3}\right)$ below $p<0.72$ and becomes positive finite above 


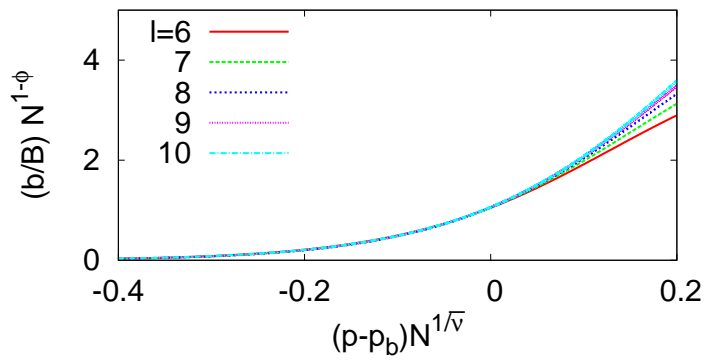

FIG. 5: (Color online) Scaling plot of $b / B$ in $\{7,3\}$ using Eq. (44), with $p_{b}=0.72, \phi=0.82$, and $1 / \bar{\nu}=0.12$.
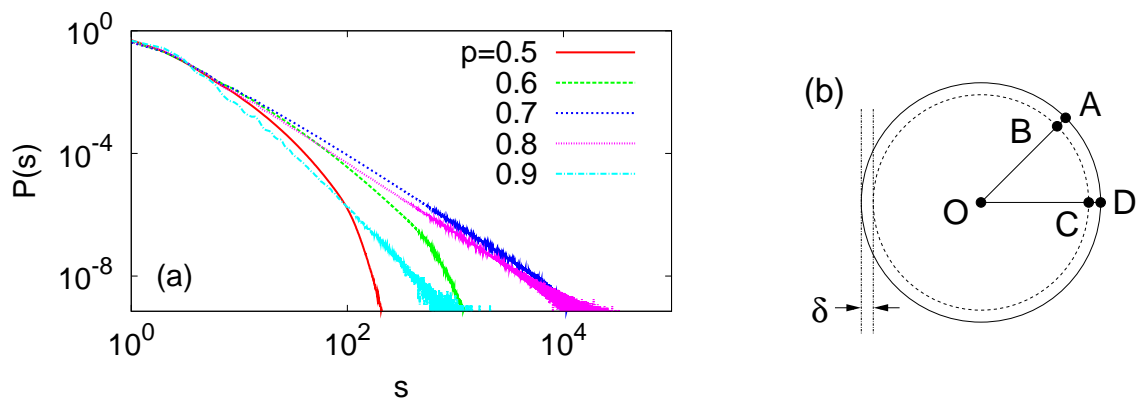

FIG. 6: (Color online) (a) Cluster size distribution, $P(s)$, at various $p$ in $\{7,3\}$. It shows a power-law form also above $p_{u}$. (b) A schematic view of the Poincaré disk centered at $O$. The lattice is constructed up to the dashed circle, which is apart from the circumference of the disk by $\overline{A B}=\overline{C D}=\delta$ in the Euclidean measure.

that, suggesting $p_{b} \approx 0.72$. It thus suggests $p_{b}=p_{u}$ within our numerical accuracy. This corresponding scaling exponent $\phi(p)$ is plotted in Fig. 4(d). While $\phi(p)$ is an increasing function of $p$ in the Cayley tree, it is a convex function in $\{7,3\}$. The crucial difference is that $\phi$ is still less than one at $p=p_{b}$. In order to study the transition at $p_{b}$, we use the finite-size scaling form:

$$
b / B \sim N^{\phi-1} f\left[\left(p-p_{b}\right) N^{1 / \bar{\nu}}\right] .
$$

The scaling collapse at $p_{b}=0.72$ determines the critical indices to $\phi \approx 0.82$ and $\bar{\nu} \approx 0.12$ (Fig. [5).

Another crucial difference from the Cayley tree is the appearance of a supercritical region at $p>p_{u}$. We find that the hyperbolic lattices in this region display a power-law behavior in the cluster size distribution [Fig. 6(a)]. A hand-waving argument for this behavior goes 

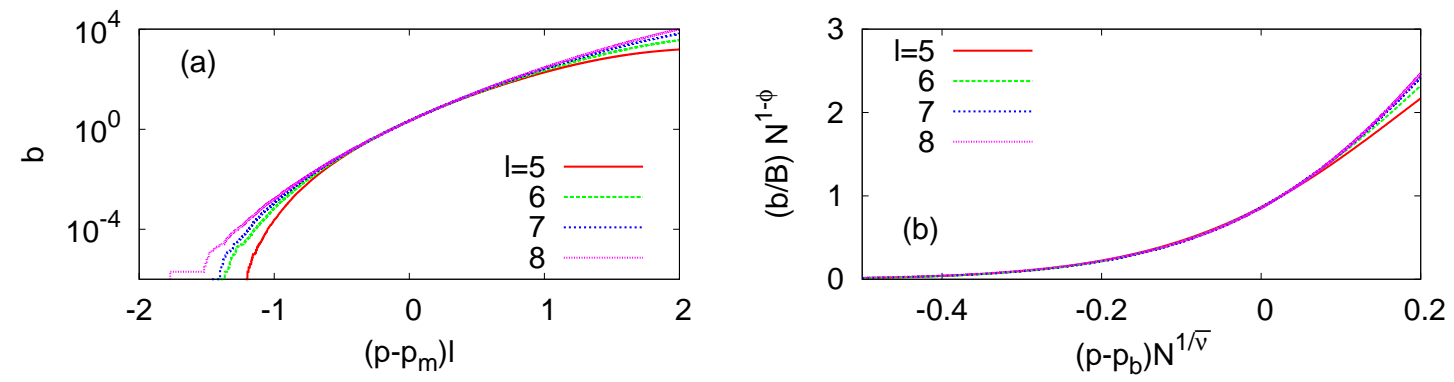

FIG. 7: (Color online) Numerical results for $\{4,5\}$, averaged over $10^{6}$ realizations. (a) Scaling plot with $p_{m}=0.27$. (b) Scaling collapse with $p_{b}=0.52, \phi=0.82$, and $1 / \bar{\nu}=0.11$.

as follows: The probability of finding a cluster with a certain size is dominated by its surface area, since connections through the surface have to be cut to isolate this cluster from the surrounding. It is thus believed that $P(s) \propto \exp \left[-\eta(p) s^{1-1 / d}\right]$ in a $d$-dimensional lattice with some $p$-dependent constant $\eta(p)[9,20]$. For a hyperbolic lattice with $d=\infty$, one may well expect $P(s) \propto e^{-s}$. In case of our nonamenable setting, however, most large-sized clusters are facing the outmost boundary of the lattice, where the outward connections are already absent. Suppose a cluster contained in a fan-shape $O B C$ on the Poincaré disk, where the lattice is constructed up to the dashed line in Fig. G(b). If $\overline{A B}=\overline{C D}=\delta$ in the Euclidean measure, the hyperbolic length of the arc $\widehat{B C}$ connecting $B$ and $C$ is of an order of $\delta^{-1}$. Since the cluster size is closely related to the surface length in a hyperbolic lattice, which is proportional to $\delta^{-1}$ [17], we may say that $s \propto \delta^{-1}$. At the same time, the hyperbolic length that one should cut out to isolate this cluster is roughly the length of the geodesic connecting $B$ and $C$, which grows as $\log \delta^{-1}$. Note that $\widehat{B C}$ needs not be considered here since it is a part of the lattice boundary, and this makes the fundamental difference from the exponential decay. In other words, the number of bonds cut for hyperbolic lattices is not proportional to the size $s$, but only to $\log s$. This gives the cluster size distribution in a power-law form as $P(s) \sim \exp [-\eta(p) \log s]=s^{-\eta(p)}$. It is also possible to infer that $\eta(p)$ should be an increasing function of $p$, as clusters are merged to the largest one in the supercritical phase. 

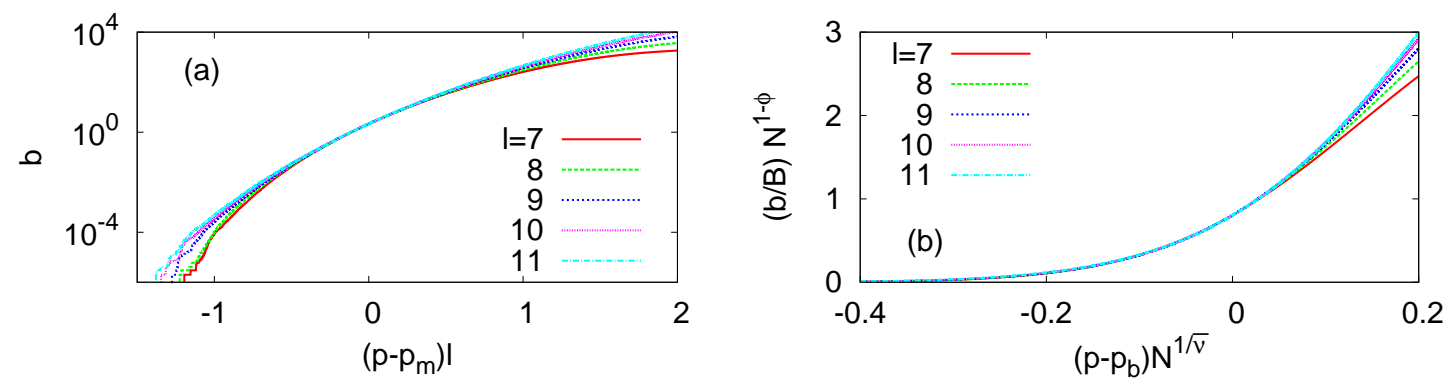

FIG. 8: (Color online) Numerical results for $\{3,7\}$, averaged over $10^{6}$ realizations. Here are shown the scaling plots (a) at $p_{m}=0.20$ and (b) at $p_{b}=0.37$ with $\phi=0.82$ and $1 / \bar{\nu}=0.11$.

\section{Comparison with $\{4,5\}$ and $\{3,7\}$}

In order to investigate the generality of our results, we also study two additional hyperbolic lattices with different structures $\{m, n\}$. In all cases we find precisely the same scenario. For the hyperbolic lattice $\{4,5\}$, we find $p_{m} \approx 0.27, p_{u}=p_{b} \approx 0.52$ [Figs. 7](a) and 7(b)]. The scaling behavior at the lower threshold $p_{m}$ is the same as for the Cayley tree and for the lattice $\{7,3\}$. At the upper threshold $p_{b}=0.52$, the critical indices $\phi=0.82$ and $1 / \nu=0.11$ was determined from the scaling collapse [Fig. [7(c)]. These values are identical to the ones found for lattice $\{7,3\}$ within numerical accuracy.

For the lattice $\{3,7\}$ which is dual to $\{7,3\}$ the same agreement is found: The same size scaling is found at the lower threshold $p_{m} \approx 0.20$ [Fig. 8(a)]. At the upper one, $p_{u} \approx 0.37$, the critical indices $\phi=0.82$ and $1 / \bar{\nu}=0.11$ are found [Fig. $8(\mathrm{~b})]$. This is again in striking agreement with the lattices $\{7,3\}$ and $\{4,5\}$. Thus our results are consistent with a universal critical behavior at the second threshold for all hyperbolic lattices $\{m, n\}$ provided both $m$ and $n$ are finite numbers. This critical behavior is distinct from the tree case $\{\infty, n\}$, which has $\phi=1$ at $p=p_{b}$. The present accuracy suggests that the critical indices are to good approximation $\phi \approx 0.82$ and $1 / \bar{\nu} \approx 0.11 \pm 0.01$.

We also note that while the lower threshold $p_{m}$ is still close to the tree result $1 /(n-1)=$ $1 / 4$ for $\{4,5\}$, the deviation becomes large for $\{3,7\}$, and that the estimate $p_{b}=p_{u}=$ $m /(m+n)$ in general only gives a very crude estimate of the upper threshold. 


\section{SUMMARY}

We have investigated the percolation thresholds and the critical properties of the percolation transitions for hyperbolic lattices using finite-size scaling methods. Two distinct percolation thresholds were found: The lower one corresponds to the threshold when the probability of finding a cluster from the midpoint to the boundary becomes finite and the second when the cluster containing the midpoint, with finite probability, also contains a finite fraction of the boundary. This is in contrast to the planar lattices which only possess a single percolation threshold because the two thresholds above coincide. The Cayley tree was used as a benchmark. It was found that the lower threshold for the hyperbolic lattices has the same scaling properties as for the Cayley tree and that the power-law dependencies characterizing the region between the two thresholds are also like Cayley trees. However, the second higher threshold has a different critical behavior. Our results are consistent with a universal behavior at the higher threshold for all hyperbolic lattices $\{m, n\}$ with $m$ and $n$ finite. This critical behavior is characterized by two critical indices $\phi \approx 0.82$ and $\nu \approx 0.11$. What actually determines these critical indices is still an open question and will be the subject of future research.

\section{Acknowledgments}

We are grateful to Bo Söderberg, Jae Dong Noh, Sang-Woo Kim, Hiroyuki Shima, and Okyu Kwon for their inspirations and help. S.K.B. and P.M. acknowledge the support from the Swedish Research Council with the Grant No. 621-2002-4135. B.J.K. was supported by the Korea Research Foundation Grant funded by the Korean Government (MOEHRD) with Grant No. KRF-2007-313-C00282. This research was conducted using the resources of High Performance Computing Center North (HPC2N).

[1] S. N. Dorogovtsev and A. V. Goltsev, Rev. Mod. Phys. 80, 1275 (2008).

[2] Y. Gefen, B. B. Mandelbrot, and A. Aharony, Phys. Rev. Lett. 45, 855 (1980); S. Havlin, D. ben-Avraham, and D. Movshovitz, ibid. 51, 2347 (1983); B. Kutnjak-Urbanc, S. Zapperi, 
S. Milošević, and H. E. Stanley, Phys. Rev. E 54, 272 (1996); H. D. Rozenfeld and D. benAvraham, ibid. 75, 061102 (2007).

[3] J. S. Andrade, H. J. Herrmann, R. F. S. Andrade, and L. R. da Silva, Phys. Rev. Lett. 94, $018702(2005)$.

[4] H. Shima and Y. Sakaniwa, J. Stat. Mech.: Theory Exp. (2006) P08017; J. Phys. A 39, 4921 (2006); S. K. Baek, P. Minnhagen, and B. J. Kim, Europhys. Lett. 79, 26002 (2007); S. K. Baek, S. D. Yi, and B. J. Kim, Phys. Rev. E 77, 022104 (2008); C. D. Modes and R. D. Kamien, Phys. Rev. Lett. 99, 235701 (2007).

[5] D. Vanderbilt and J. Tersoff, Phys. Rev. Lett. 68, 511 (1992); N. Park, M. Yoon, S. Berber, J. Ihm, E. Osawa, and D. Tománek, ibid. 91, 237204 (2003).

[6] R. H. Schonmann, in Perplexing Probability Problems: Papers in Honor of Harry Kesten, edited by M. Bramson and R. Durrett, (Birkhäuser, Boston, 1999), pp. 53-67.

[7] I. Benjamini and O. Schramm, Ann. Probab. 27, 1347 (1999); J. Am. Math. Soc. 14, 487 (2001).

[8] R. Lyons, J. Math. Phys. 41, 1099 (2000).

[9] D. Stauffer and A. Aharony, Introduction to Percolation Theory, 2nd ed. (Taylor \& Francis, London, 2003).

[10] R. H. Schonmann, Commun. Math. Phys. 219, 271 (2001); ibid. 225, 453 (2002).

[11] M. E. J. Newman and R. M. Ziff, Phys. Rev. Lett. 85, 4104 (2000); Phys. Rev. E 64, 016706 (2001).

[12] A. Zen, A. Kabakçığlu, and A. L. Stella, Phys. Rev. E 76, 026110 (2007).

[13] B. Sapoval, M. Rosso, and J. F. Gouyet, J. Phys. (France) Lett. 46, L149 (1985).

[14] S. K. Baek, P. Minnhagen, and B. J. Kim (unpublished).

[15] H. S. M. Coxeter, M. S. Longuet-Higgins, and J. C. P. Miller, Philos. Trans. R. Soc. London, Ser. A 246, 401 (1954).

[16] H. S. M. Coxeter, Can. Math. Bull. 40, 158 (1997).

[17] J. W. Anderson, Hyperbolic Geometry (Springer, London, 1999).

[18] B. Söderberg, Phys. Rev. E 47, 4582 (1993).

[19] P. J. Flory, J. Am. Chem. Soc. 63, 3083 (1941).

[20] G. Grimmett, Percolation (Springer, New York, 1989).

[21] S. K. Baek, Hoang Anh Tuan Kiet, and B. J. Kim, Phys. Rev. E 76, 046113 (2007). 
[22] A. G. Hunt, Percolation Theory for Flow in Porous Media (Springer, Berlin, 2005). 